DOI: 10.12731/wsd-2018-4-189-203

УДК 616.1-056.257(571.1/.5).

\title{
ПОРОГОВЫЕ ЗНАЧЕНИЯ ОКРУЖНОСТИ ТАЛИИ, ОПРЕДЕЛЯЮЩИЕ РИСК МЕТАБОЛИЧЕСКОГО СИНДРОМА У МУЖЧИН И ЖЕНЩИН РЕСПУБЛИКИ ХАКАСИЯ
}

\author{
Берсенёва О.А., Агеева Е.С.
}

Цель исследования - определить пороговые значения окружности талии для идентификации метаболического синдрома среди населения Республики Хакасия.

Материал и методы. Обследовано 300 пациентов с метаболическим синдромом (50 (42-54) лет). Группу контроля составили 100 здоровых доноров (43 (36-47) лет). Обследованные были представлены 2 группами -хакасами и европеоидами, проживающими в Республике Хакасия.

Включение пачиентов в исследование осуществлялось с учетом критериев метаболического синдрома JIS (2009). Оиенивали антропометрические показатели, артериальное давление, уровни холестерина, липопротеинов высокой и низкой плотности, триглицеридов, гликемии натощзак. Статистическую обработку результатов исследования проводили с использованием пакета «BioStat».

Результаты. В ходе исследования получены результаты порогового значения окружности талии для идентификации метаболического синдрома. Для определения метаболического синдрома у женщин хакасок точка разделения Cut-off составила 74 cм (Se - 98,8\%, Sp-93,75\%), площадь под кривой $A U C-0,99$, у женщин-европеоидок- 84 см (Se - 100\%, $S p-100 \%)$, площадь под кривой AUC-0,97. Пороговое значение окружности талии для мужчин хакасов с метаболическим синдромом составило $92 \mathrm{cм}(\mathrm{Se}-100 \%, \mathrm{Sp}-100 \%)$, площчадь под кривой AUC - 1,0, у мужчин-европеоидов - 96 см (Se-100\%, Sp-100\%), площадь под кривой $A U C-0,99$.

Заключение. Таким образом, у пациентов с компонентами метаболического синдрома были определены пороговые значения окружности талии для идентификации метаболического синдрома. Полученные результаты свидетельствуют о наличии этнических различий в патогенезе метаболических нарушений у мужчин и женщиин Республики Хакасия. 
Ключевые слова: метаболический синдром; пороговые значения окружности талии, абдоминальное ожирение.

\title{
THE CUT-OFF POINTS OF WAIST CIRCUMFERENCE FOR DETERMINATION OF RISK OF METABOLIC SYNDROME AMONG MALES AND FEMALES IN REPUBLIC OF KHAKASSIA
}

\author{
Bersenyeva O.A., Ageeva E.S.
}

Background. The aim of this study was to determine the cut-off points of waist circumference for identification of metabolic syndrome among populations by the Republic of Khakassia.

Materials and methods. 300 patients with metabolic syndrome (aged 50 (42-54) years) were investigated. The control group was of 100 healthy donors (43 (36-47) years). All participants were presented by 2 groups - populations of Khakas and Caucasian has been lived in the Republic of Khakassia.

The patients who had components of metabolic syndrome by recommendation JIS (2009) were included to research. In the study were evaluated anthropometric characteristics, blood pressure, cholesterine, cholesterol, high-density and low-density lipoprotein cholesterol, triglycerides and glucose levels. Statistical analysis was performed using the application package «BioStat».

Results: Our analysis revealed that cut-off values of waist circumference for identification of metabolic syndrome in females Khakas with metabolic syndrome consist of cut-off values of $74 \mathrm{~cm}$ ( $\mathrm{Se}-98,8 \%, \mathrm{Sp}-93,75 \%)$, area under the curve AUC-0,99, in females Caucasian - $84 \mathrm{~cm}(\mathrm{Se}-100 \%, \mathrm{Sp}-$ $100 \%)$, area under the curve AUC-0,97. Cut-off values of cut-off values of waist circumference in males Khakas with metabolic syndrome consist of 92 $\mathrm{cm}$ (Se-100\%, Sp-100\%), area under the curve AUC-1,0, in males Caucasian-96 cm (Se-100\%, Sp-100\%), area under the curve AUC-0,99.

Conclusion: thus, from patients with components of metabolic syndrome have been determined cut-off values of waist circumference for identification of metabolic syndrome. The findings showed are ethnic features of pathogenesis of metabolic disorders from males and females in Republic of Khakassia.

Keywords: metabolic syndrome; cut-off values of waist circumference, abdomen obesity. 
Метаболический синдром представляет собой комплекс обменных нарушений (абдоминальное ожирение, артериальная гипертензия, дислипидемия атерогенного профиля, в частности гипертриглицеридемия и снижение уровня ХС ЛПВП, нарушения углеводного обмена от НТГ до СД 2 типа и др.), этиопатогенетически связанных между собой и ускоряющих развитие и прогрессирование атеросклеротических сердечнососудистых заболеваний, сахарного диабета 2 типа [1, с. 109-115; 2, с. 55-65; 3, c. $1050-1067 ; 4$, C. $25 ; 5$, с. 1-7].

В Декларации IDF (2005), в консенсусе по метаболическому синдромy JIS (2009) определена актуальность исследований по изучению особенностей МС в различных этнических группах с целью дополнения и уточнения критериев метаболического синдрома [6, с. е 0148976; 7, c. 429].

Ряд исследований отмечает наличие этнических особенностей не только в распространенности МC, но и его отдельных компонентов так в США доминирующим компонентом служит ожирение, в Европе - атерогенная дислипидемия, а в афро-американских странах - АГ [8, с. 15-33; 9, с. $34-42 ; 10$, с. 2014].

Для азиатской популяции в отличие от европейской, характерно наличие ИР при меньших показателях ИМТ и ОТ [11, с. 1; 12, с. 154-166].

В связи с этим можно предположить, что для диагностики метаболического синдрома в каждой популяций необходимо использовать свои критерии с учетом региональных особенностей. Между тем, Республика Хакасия отличается многонациональным составом населения, что вызывает большой интерес в плане изучения метаболического синдрома и его компонентов.

Цель исследования - определить пороговые значения окружности талии для идентификации метаболического синдрома у населения Республики Хакасия.

\section{Материалы и методы}

Исследование проведено у 200 женщин и 100 мужчин с метаболическим синдромом, из них европеоидов - 150 чел., хакасов - 150 чел., находившихся на обследовании и лечении в ГБУЗ РХ «РКБ им. Г.Я. Ремишевской». Средний возраст пациентов с метаболическим синдромом составил 50 (42-54) лет. Контрольную группу составили здоровые доноры из них 50 европеоидов и 50 хакасов (50 женщин и 50 мужчин). Возраст обследованных в контрольной группе - 43 (36-47) лет. Все обследован- 
ные - коренные (хакасы или монголоиды) и пришлые жители Республики Хакасия (русские или европеоиды).

Включение пациентов в исследование осуществлялось с учетом критериев метаболического синдрома JIS (2009) для различных этнических групп, которые включают три и более любых из пяти компонентов: абдоминальное ожирение - для азиатской группы при ОТ $\geq 90$ см у мужчин и $\geq 80$ см у женщин, абдоминальное ожирение - для европеоидной группы при ОТ $\geq 94$ см у мужчин и $\geq 80$ см у женщин; уровень систолического артериального давления $\geq 130$ мм рт. ст. и/или диастолическое артериальное давление $\geq 85$ мм рт. ст. или терапия артериальной гипертонии; уровень триглицеридов $\geq 1,7$ ммоль/л или терапия дислипидемии; уровень липопротеинов высокой плотности у женщин $<1,3$ ммоль/л, у мужчин $<1,0$ ммоль/л или терапия дислипидемии; уровень гликемии натощак $\geq 5,6$ ммоль/л или сахароснижающая терапия [13, с. 1640-1645].

Критериями исключения из исследования являлись: наличие тяжелых соматических, онкологических, гематологических, эндогенных психических заболеваний; отсутствие согласия принимать участие в исследовании.

Всем больным проведено комплексное клинико-лабораторное исследование: определялись антропометрические показатели, проводился биохимический анализ крови, который включал определение уровней общего холестерина (ХC), липопротеинов высокой плотности (ЛПВП), липопротеинов низкой плотности (ЛПНП), триглицеридов (ТГ), уровня гликемии натощак.

Статистическая обработка результатов исследования проводилась с использованием пакета статистических программ «BioStat». Для проверки гипотезы о нормальности распределения использовался критерий Колмогорова-Смирнова. Учитывая, что все данные имели распределения, отличные от нормального, вычисления средних значений представлены в виде медианы (Ме) и интерквартильного размаха (Q25 и Q75). При анализе различий значений был использован критерий Манна-Уитни. Различия считались статистически значимыми при $p<0,05$. Для идентификации наличия/ отсутствия метаболического синдрома показатели компонентов метаболического синдрома определялись с помощью ROC-модели - расчет оптимального (порогового) значения окружности талии. Критерием выбора порога отсечения взято требование максимальной суммарной чувствительности и специфичности модели: Cut-off $=\max (\mathrm{Se}+\mathrm{Sp})$, где $\mathrm{Se}-$ чувствительность, $\mathrm{Sp}$ - специфичность. 


\section{Результаты и обсуждения}

По дефинициям IDF (2005), распространенность МС в общей популяции составляет 51,9\%, у коренных (43\%) ниже, чем у некоренных $66,2 \%, \mathrm{p}<0,001$. По дефинициям JIS (2009), распространенность МС в общей популяции составляет 55\%, у коренных - 56\%, что достоверно ниже, чем в некоренной популяции - 63\%. Таким образом, распространенность MC среди хакасов с применением критериев JIS у хакасов увеличилась на $15 \%$. Назначение абдоминального ожирения как важного, а не необязательного компонента для диагностики МС не позволяет идентифицировать долю людей с метаболическим синдромом.

Европеоиды с метаболическим синдромом имеют более высокие показатели индекса массы тела, чем хакасы: $31,9(29,7-34,2)$ против 30,1 $(28,2-32,6)$ кг/м2, $<<0,001$. В результате исследования антропометрических показателей было выявлено, что у европеоидов ОТ составил 109,0 $(92,5-119,0)$ см, что было статистически значимо выше, по сравнению с ОТ у хакасов $(99,0(92,0-102,0) \mathrm{cm}, \mathrm{p}=0,017)$. Такая же тенденция отмечалась и для показателя ОБ: у европеоидов - 107,0 $(99,5116,0)$ см, у хакасов - 102,0 $(98,0-104,0)$ см. Хотя различия не имели статистически значимого характера $(\mathrm{p}>0,05)$. Анализ корреляции ОТ у пациентов с МС выявил достоверные взаимосвязи с показателями липидного и углеводного обмена, как у монголоидов, так и у европеоидов. Так у женщин хакасок выявлены достоверные взаимосвязи с ОХС, ТГ, ЛПОНП ( $\mathrm{r}=-0,4)$, и глюкозой $(\mathrm{r}=-0,5), \mathrm{p}<0,001$. Аналогичная взаимосвязь наблюдалась и среди женщин европеоидок. Кроме того в данной группе исследуемых выявлена взаимосвязь ОТ с показателями систолического и диастолического артериального давления у $(\mathrm{r}=-0,4), \mathrm{p}<0,001$. В группах мужчин выявлены достоверные взаимосвязи ОТ только с уровнем диастолического артериального давления, как среди монголоидов, так и европеоидов $(\mathrm{r}=-0,42$; $\mathrm{p}=0,046)$.

У европеоидов с метаболическим синдромом, по сравнению с хакасами, увеличивается масса жировой ткани 23,66 (20,0-28,7) против 26,2 $(21,6-31,0)$ кг, $\mathrm{p}<0,001$, объем общей жировой ткани 25,6 $(21,7-31,1)$ против 28,4 $(23,4-33,6)$ л, $\mathrm{p}<0,001$, объем подкожной жировой ткани 17,15 $(13,5-21,8)$ против $20,76(15,626,7)$ л, $\mathrm{p}<0,001$, в то время как у хакасов преобладает объем висцеральной жировой ткани $8,9(7,5-9,6)$ против 7,5 $(4,5-9,6)$ л, $\mathrm{p} \geq 0,05$.

Накопление объема общего жира в организме происходит в результате отложения жира в подкожных и висцеральных депо. На начальном этапе 
преимущественно увеличивается подкожное депо. Однако в какой-то момент происходит диспропорция накопления жировой ткани с преимущественным отложением в висцеральных депо [14, с. 3-8]. Это позволяет предположить, что у хакасов диспропорция накопления жировой ткани является более выраженной и находит свое выражение при меньших антропометрических параметрах. Параметры липидного и углеводного обмена в двух этнических группах в зависимости от пола представлены в таблице 1.

Таблица 1.

Показатели липидного и углеводного обмена в обследованных этнических группах в зависимости от пола $\left(\mathrm{Me}, \mathrm{Q}_{25}-\mathrm{Q}_{75}\right)$

\begin{tabular}{|c|c|c|c|c|c|c|c|c|}
\hline \multirow{3}{*}{ Показатели } & \multicolumn{4}{|c|}{$\begin{array}{c}\text { Монголоиды с } \\
\text { метаболическим синдромом }\end{array}$} & \multicolumn{4}{|c|}{$\begin{array}{c}\text { Европеоиды с } \\
\text { метаболическим синдромом }\end{array}$} \\
\hline & \multicolumn{2}{|c|}{$\begin{array}{c}\text { Мужчины, } \\
\text { N=50 (1) }\end{array}$} & \multicolumn{2}{|c|}{$\begin{array}{c}\text { Женщины, } \\
\mathrm{N}=100 \text { (2) }\end{array}$} & \multicolumn{2}{|c|}{$\begin{array}{c}\text { Мужчины, } \\
\mathrm{N}=50 \text { (3) }\end{array}$} & \multicolumn{2}{|c|}{\begin{tabular}{|c|} 
Женщины, \\
N=100 (4)
\end{tabular}} \\
\hline & $\mathrm{Me}$ & Q25-Q75 & $\mathrm{Me}$ & Q25-Q75 & $\mathrm{Me}$ & Q25-Q75 & $\mathrm{Me}$ & Q25-Q75 \\
\hline \multirow{2}{*}{$\begin{array}{l}\text { Общий } \\
\text { холестерин, } \\
\text { ммоль/л }\end{array}$} & 6,4 & & 5,7 & & 6,2 & & 5,9 & \\
\hline & \multicolumn{2}{|c|}{$P_{1-2}-0,018$} & \multicolumn{2}{|c|}{$P_{2-4}-0,079$} & \multicolumn{2}{|c|}{$P_{1-3}-0,484$} & \multicolumn{2}{|c|}{$P_{3-4}-0,274$} \\
\hline \multirow{2}{*}{$\begin{array}{l}\text { ХС-ЛПНП, } \\
\text { ммоль/л }\end{array}$} & 3,4 & $2,8-4,6$ & 3,5 & $2,8-4,6$ & 3,8 & $2,7-4,7$ & 3,5 & $2,8-4,5$ \\
\hline & \multicolumn{2}{|c|}{$P_{1-2}-0,858$} & \multicolumn{2}{|c|}{$P_{2-4}-0,949$} & \multicolumn{2}{|c|}{$P_{1-3}-0,338$} & \multicolumn{2}{|c|}{$\mathrm{P}_{3-4}-0,323$} \\
\hline \multirow{2}{*}{$\begin{array}{l}\text { ХС-ЛПВП, } \\
\text { ммоль/л }\end{array}$} & 0,99 & $0,82-1,26$ & 1,15 & $1,01-1,31$ & \begin{tabular}{|l|}
1,02 \\
\end{tabular} & $0,88-1,29$ & 1,18 & $0,96-1,59$ \\
\hline & \multicolumn{2}{|c|}{$P_{1,-0,019}$} & \multicolumn{2}{|c|}{$P_{2-4}-0,576$} & \multicolumn{2}{|c|}{$P_{1-3}-0,490$} & \multicolumn{2}{|c|}{$P_{3-4}-0,012$} \\
\hline \multirow{2}{*}{$\begin{array}{l}\text { Триглицериды, } \\
\text { ммоль/л }\end{array}$} & 2,3 & $1,96-3,18$ & 2,0 & $1,5-2,7$ & 2,4 & $1,78-3,2$ & 2,0 & $1,3-2,8$ \\
\hline & \multicolumn{2}{|c|}{$P,-0,048$} & \multicolumn{2}{|c|}{$P_{2-4}-0,983$} & \multicolumn{2}{|c|}{$P_{1-3}-0,942$} & \multicolumn{2}{|c|}{$P_{3-4}-0,014$} \\
\hline \multirow{2}{*}{$\begin{array}{l}\text { ХС-ЛПОНП, } \\
\text { ммоль/л }\end{array}$} & 1,04 & $0,9-1,43$ & 0,90 & $0,68-1,22$ & \begin{tabular}{|l|}
1,09 \\
\end{tabular} & $0,80-1,45$ & 0,90 & $0,59-1,28$ \\
\hline & \multicolumn{2}{|c|}{$P_{1-2}-0,052$} & \multicolumn{2}{|c|}{$P_{2-4}-0,985$} & \multicolumn{2}{|c|}{$P_{1-3}-0,937$} & \multicolumn{2}{|c|}{$P_{3-4}-0,016$} \\
\hline \multirow{2}{*}{$\begin{array}{l}\text { Индекс } \\
\text { атерогенности, } \\
\text { у.е. }\end{array}$} & 5,2 & $3,5-7,26$ & 3,72 & $2,5-4,96$ & 4,65 & $3,46-6,52$ & 3,83 & $2,36-5,17$ \\
\hline & \multicolumn{2}{|c|}{$P_{1-2}-0,003$} & \multicolumn{2}{|c|}{$\mathrm{P}_{2-4}-0,817$} & \multicolumn{2}{|c|}{$P_{1-3}-0,412$} & & $4^{-0,007}$ \\
\hline & 7,8 & $6,3-10,5$ & 7,2 & $5,46-9,2$ & 8,5 & $6,3-10,9$ & 7,0 & $5,2-11,9$ \\
\hline & & $-0,388$ & & ${ }_{4}^{-0,557}$ & & ${ }_{3}-0,336$ & & $4^{-0,192}$ \\
\hline
\end{tabular}

Примечание: $\mathrm{p}_{1-2}$ - достоверные различия между мужчинами и женщинами монголоидами с MC; $\mathrm{p}_{1-3}$ - достоверные различия между мужчинами монголоидами и европеоидами с $\mathrm{MC} ; \mathrm{p}_{2-4}$-достоверные различия между женщинами монголоидами и европеоидами с $\mathrm{MC} ; \mathrm{p}_{3-4}$ - достоверные различия между женщинами монголоидами и европеоидами с МС. 
Как следует из таблицы 1, содержание общего холестерина, как у монголоидов, так и европеоидов (мужчин и женщин) незначительно превышало нормативные значения. Данный показатель у женщин-европеоидок, был несколько выше, чем у женщин-монголоидов, в то время как в группе мужчин данный показатель был выше среди монголоидов.

Аналогичные данные получены при обследовании коренного населения Мордовия: более высокие уровни ОХС у монголоидов (мокшан) по сравнению с европеоидами (русскими), что свидетельствует об этнических различиях детерминации особенностей патогенеза метаболических нарушений [15, с. 97-101].

Аналогичная тенденция в липидном спектре наблюдалась и в содержании ХС ЛПНП и ТГ, что, несомненно, говорит о наличии, как у монголоидов, так и у европеоидов гиперхолестеринемии и гипертриглицеридемии: при этом у мужчин выше, чем у женщин. Наблюдается тенденция к увеличению данных показателей среди мужчин европеоидов. Обращает на себя внимание совершенно противоположная направленность динамики содержания ХС ЛПВП и ТГ-богатых ЛПОНП у мужчин - как монголоидов, так и европеоидов. Несмотря на то, что у мужчин европеоидов уровень ХС ЛПВП не выходил за пределы референсных значений он сочетался с высокими значениями ТГ и ХС ЛПОНП. У мужчин монголоидов низкие значения ХС ЛПВП сочетались с высоким содержанием ТГ и ХС ЛПОНП. Аналогичная тенденция наблюдалась среди женщин монголоидов и европеоидов. Обращает на себя внимание более высокий уровень ХС ЛПНП у мужчин европеоидов.

По данным литературы среднее значение ХСЛПВП у монголоидов (ненцев) было выше нормы (1,23 ммоль/л у женщин) и даже выше (1,36 ммоль/л у мужчин), что, несколько отличается от полученных нами результатов и может свидетельствовать о формировании у монголоидов неблагоприятного липидного профиля [16, с. 67-69].

Атерогенное соотношение отдельных компонентов липидного спектра у представителей обоего пола монголоидов и европеоидов подтверждает и расчет индекса атерогенности, величина которого была максимальна у коренных мужчин.

Содержание глюкозы у представителей, как монголоидов, так и европеоидов (мужчин и женщин) превышал референсные значения. Данный показатель у европеоидов, был несколько выше, чем у монголоидов. Полученные нами результаты согласуются с данными, полученными при обследовании коренного населения Горной Шории [17, с. 108-111]. 
Медианы показателей липидного и углеводного обмена превышали нормативные величины в исследуемых группах, но статистически значимых различий по полу ни для одного из изучаемых показателей не получено (p>0,05).

В ходе исследования получены результаты порогового значения окружности талии для идентификации метаболического синдрома. По данным ROC-анализа точка разделения (Cut-off) для определения субъектов с метаболическим синдромом у женщин хакасок составила 74 см ( $\mathrm{Se}-98,8 \%, \mathrm{Sp}-93,75 \%)$, площадь под кривой $\mathrm{AUC}-0,99$, у русских женщин $84 \mathrm{~cm}(\mathrm{Se}-100 \%, \mathrm{Sp}-100 \%)$, площадь под кривой $\mathrm{AUC}-1,0$. Пороговое значение окружности талии (Cut-off) для определения субъектов с метаболическим синдромом мужчин хакасов составило 92см ( $\mathrm{Se}-100 \%, \mathrm{Sp}-100 \%)$, площадь под кривой $\mathrm{AUC}-1,0$, у русских мужчин 96см ( $\mathrm{Se}-100 \%, \mathrm{Sp}-100 \%)$, площадь под кривой AUC - 0,99 (Рис. 1).

Важно отметить, что рассчитанные нами отрезные (пороговые) значения ОТ для мужчин монголоидов составили 92 см, что выше, чем в критериях WPRO для жителей Азии (>90 cм) и практически совпадает с критерием АО для мужчин-европеоидов (> 94 см) [18, с. 172-177].

При этом ОТ у мужчин монголоидов в кыргызской этнической группе составляла $\geq 94$ см у мужчин $[19$, с. $1-6]$.

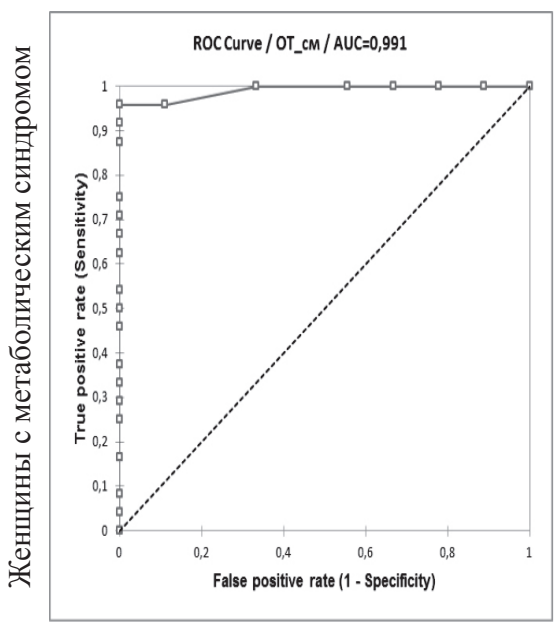

Монголоиды

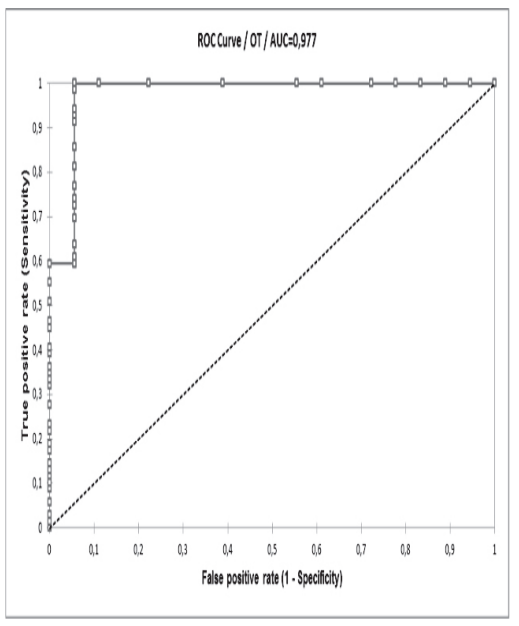

Европеоиды 


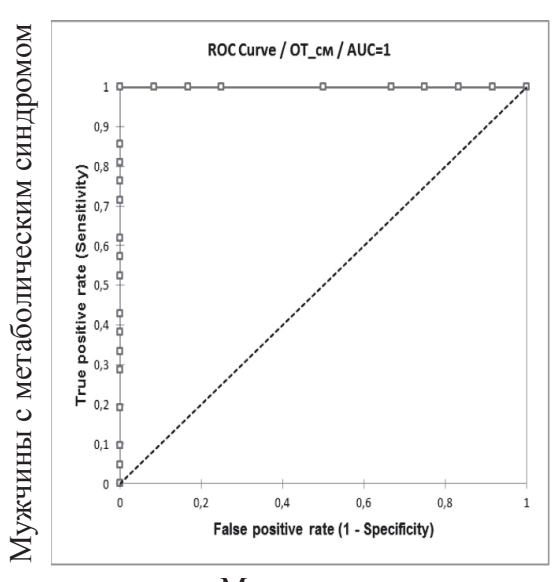

Монголоиды

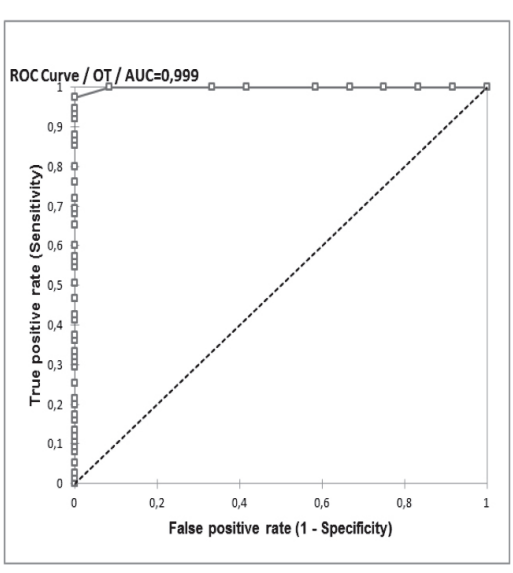

Европеоиды

Рис. 1. Чувствительность и специфичность, обнаружение трех критериев метаболического синдрома при увеличении окружности талии у женщин и мужчин Республики Хакасия (ROC кривая)

Полученные нами результаты были близки к результатам исследования Т.М. Климовой, в Якутске, где у мужчин пороговое значение ОТ составило $\geq 88,6$ см [20, с. 172-177]. Показатели ОТ для компонентов МС у женщин монголоидов, составили 77 см и были ниже пороговых значений для женщин азиаток [21, с. 209-216]. По литературным данным, отрезные точки ОТ у женщин, близкие по показателям к нашим, выявлены в Японии - 74 см [22, с. 474-481]. При этом на Среднем Востоке в 2007 г. составили - 91 см, определенные в Ираке - 99 см, в Малой Азии у женщин были от 83-88 см ,в Китае, Канаде. и в Корее, отрезная точка ОТ для компонентов МС составила 80 см [23, с. 1092-1097]. Возможно, данный феномен объясняется тем, что у коренных народов Сибири наблюдаются особенности морфологических и биохимических факторов. Среди которых развитие метаболических нарушений при меньших габаритных размерах тела [24, с. 71-74].

\section{Заключение}

Таким образом, в популяции коренных жителей Республики Хакасия для идентификации лиц с наличием $\geq 3$ компонентов метаболического синдрома пороговое значение окружности талии составило 92 см для мужчин и 74 см для женщин. При этом в популяции пришлых жителей 
республики Хакасия для идентификации лиц с наличием $\geq 3$ компонентов метаболического синдрома пороговое значение окружности талии составило 96 см для мужчин и 84 см для женщин. Полученные результаты свидетельствуют об этнических различиях детерминации особенностей патогенеза метаболических нарушений

\section{Список литературы}

1. Bonomini F., Rodella L.F., Rezzani R. Metabolic syndrome, aging and involvement of oxidative stress. Aging and disease, 2015, Vol. 6. №. 2, pp. 109-115.

2. Asrih M., Jornayvaz F.R. Metabolic syndrome and nonalcoholic fatty liver disease: Is insulin resistance the link? Molecular and cellular endocrinology, 2015, vol. 418, pp. 55-65.

3. Sperling L. S. et al. The CardioMetabolic Health Alliance Working Toward a New Care Model for the Metabolic Syndrome. Journal of the American College of Cardiology, 2015, vol. 66. №. 9, pp. 1050-1067.

4. Srikanthan K. et al. Systematic review of metabolic syndrome biomarkers: a panel for early detection, management, and risk stratification in the West Virginian population. International journal of medical sciences, 2016. vol. 13. №. 1, pp. 25.

5. Walther G. et al. Metabolic syndrome individuals with and without type 2 diabetes mellitus present generalized vascular dysfunction: cross-sectional study. Arteriosclerosis, thrombosis, and vascular biology, 2015, ATVBAHA. 114.304591, pp. 1-7.

6. Guo H. et al. The Prevalence of Metabolic Syndrome Using Three Different Diagnostic Criteria among Low Earning Nomadic Kazakhs in the Far Northwest of China: New Cut-Off Points of Waist Circumference to Diagnose MetS and Its Implications. PloS one, 2016. vol.11. №. 2, pp. e 0148976.

7. Ali O. et al. Determination of metabolic syndrome among transitional community-comparison between orang asli and malay rural area in perak . Advancing inclusive rural development and transformation in a challenging environment, 2016, pp. 429.

8. Rochlani Y. et al. Gender Differences in Metabolic Syndrome. Gender Differences in the Pathogenesis and Management of Heart Disease. Springer, Cham, 2018, pp. 15-33.

9. Pucci G. et al. Sex-and gender-related prevalence, cardiovascular risk and therapeutic approach in metabolic syndrome: A review of the literature. Pharmacological research, 2017. Vol.120, pp. 34-42.

10. Rodea-Montero E.R., Evia-Viscarra M.L., Apolinar-Jiménez E. Waist-to-height ratio is a better anthropometric index than waist circumference and BMI in pre- 
dicting metabolic syndrome among obese Mexican adolescents. International journal of endocrinology, 2014. vol. 2014.

11. Jia A. et al. Body fat percentage cutoffs for risk of cardiometabolic abnormalities in the Chinese adult population: a nationwide study. European journal of clinical nutrition, 2018, pp 1.

12. Mc Connell-Nzunga J. et al. Classification of obesity varies between body mass index and direct measures of body fat in boys and girls of Asian and European ancestry. Measurement in Physical Education and Exercise Science, 2018. Vol.22. № 2, pp. 154-166.

13. Alberti K.G. et al. Harmonizing the metabolic syndrome: a joint interim statement of the International Diabetes Federation Task Force on Epidemiology and Prevention; National Heart, Lung, and Blood Institute; American Heart Association; World Heart Federation; International Atherosclerosis Society; and International Association for the Study of Obesity. Circulation, 2009. Vol. 20, pp. 1640-1645. doi: 10.1161/Circulationaha.109.192644. Epub 2009 Oct 5 .

14. Бородкина Д.А. и др. Распределение жировых отложений: разгадка кажущегося парадокса ожирения в кардиологии? //Ожирение и метаболизм. 2017. T. 14, №. 2 . C. $3-8$.

15. Гончарова Н.Г. и др. Распространенность факторов риска артериальной гипертонии среди коренного населения Республики Мордовия // Казанский медицинский журнал. 2010. Т. 91, №1. С. 97-101.

16. Поляков Л.М. и др. Липидный спектр сыворотки крови у представителей коренного и пришлого населения ямало-ненецкого автономного округа // Сибирский научный медицинский журнал. 2015. № 6. С. 67-69.

17. Огарков М.Ю. и др. Распространенность компонентов метаболического синдрома у коренного и некоренного населения Горной Шории // Бюллетень СО РАМН. 2004. №1. С. 108-111.

18. Odegaard J.I., Chawla A. Pleiotropic Actions of Insulin Resistance and Inflammation in Metabolic Homeostasis. Science, 2013. Vol. 339, № 6116, pp. 172 177. doi: $10.1126 /$ science. 1230721 .

19. Cut off values for abdominal obesity as a criterion of metabolic syndrome in an ethnic Kyrgyz population (Central Asian region). Cardiovasc Diabetol. 2012 Feb 22;11:16. doi: 10.1186/1475-2840-11-16. DOI: 10.1186/1475-2840-11-16.

20. Климова Т.М., Федорова В.И., Балтахинова М.Е. Критерии ожирения для идентификации метаболических факторов риска у коренного сельского населения Якутии //Сибирский медицинский журнал (Иркутск). 2012. T. 115, №. 8. C. 110-113. 
21. Zaher Z.M.M. et al. Optimal cut-off levels to define obesity: body mass index and waist circumference, and their relationship to cardiovascular disease, dyslipidaemia, hypertension and diabetes in Malaysia //Asia Pacific journal of clinical nutrition. 2009. Vol , 18, №. 2, pp. 209-216.

22. Oka R. et al. Reassessment of the cutoff values of waist circumference and visceral fat area for identifying Japanese subjects at risk for the metabolic syndrome // Diabetes research and clinical practice. 2008. Vol. 79, №. 3, pp. 474-481.

23. Delavari A. et al. First nationwide study of the prevalence of the metabolic syndrome and optimal cutoff points of waist circumference in the Middle East: the national survey of risk factors for noncommunicable diseases of Iran // Diabetes care. 2009. Vol. 32, №. 6, pp. 1092-1097.

24. Берсенева О.А., Агеева Е.С. Особенности метаболического синдрома в период менопаузы у женщин Хакасии // Сибирское медицинское обозрение. 2014. № 5. С. 71-74.

\section{References}

1. Bonomini F., Rodella L.F., Rezzani R. Metabolic syndrome, aging and involvement of oxidative stress. Aging and disease, 2015, vol. 6. №. 2, pp. 109-115.

2. Asrih M., Jornayvaz F.R. Metabolic syndrome and nonalcoholic fatty liver disease: Is insulin resistance the link? Molecular and cellular endocrinology, 2015, vol.418, pp. 55-65.

3. Sperling L.S. et al. The CardioMetabolic Health Alliance Working Toward a New Care Model for the Metabolic Syndrome. Journal of the American College of Cardiology, 2015, vol. 66. №. 9, pp. 1050-1067.

4. Srikanthan K. et al. Systematic review of metabolic syndrome biomarkers: a panel for early detection, management, and risk stratification in the West Virginian population. International journal of medical sciences, 2016. vol. 13. №. 1, pp. 25.

5. Walther G. et al. Metabolic syndrome individuals with and without type 2 diabetes mellitus present generalized vascular dysfunction: cross-sectional study. Arteriosclerosis, thrombosis, and vascular biology, 2015, ATVBAHA. 114.304591, pp. 1-7.

6. Guo H. et al. The Prevalence of Metabolic Syndrome Using Three Different Diagnostic Criteria among Low Earning Nomadic Kazakhs in the Far Northwest of China: New Cut-Off Points of Waist Circumference to Diagnose MetS and Its Implications. PloS one, 2016. vol.11. №. 2, pp. e 0148976.

7. Ali O. et al. Determination of metabolic syndrome among transitional community-comparison between orang asli and malay rural area in perak. Advancing 
inclusive rural development and transformation in a challenging environment, 2016, pp. 429.

8. Rochlani Y. et al. Gender Differences in Metabolic Syndrome. Gender Differences in the Pathogenesis and Management of Heart Disease. Springer, Cham, 2018, pp. 15-33.

9. Pucci G. et al. Sex-and gender-related prevalence, cardiovascular risk and therapeutic approach in metabolic syndrome: A review of the literature. Pharmacological research, 2017. Vol.120, pp. 34-42.

10. Rodea-Montero E. R., Evia-Viscarra M. L., Apolinar-Jiménez E. Waist-toheight ratio is a better anthropometric index than waist circumference and BMI in predicting metabolic syndrome among obese Mexican adolescents. International journal of endocrinology, 2014. vol. 2014.

11. Jia A. et al. Body fat percentage cutoffs for risk of cardiometabolic abnormalities in the Chinese adult population: a nationwide study. European journal of clinical nutrition, 2018, pp. 1.

12. Mc Connell-Nzunga J. et al. Classification of obesity varies between body mass index and direct measures of body fat in boys and girls of Asian and $\mathrm{Eu}-$ ropean ancestry. Measurement in Physical Education and Exercise Science, 2018. Vol. 22. №. 2, pp. 154-166.

13. Alberti K. G. et al. Harmonizing the metabolic syndrome: a joint interim statement of the International Diabetes Federation Task Force on Epidemiology and Prevention; National Heart, Lung, and Blood Institute; American Heart Association; World Heart Federation; International Atherosclerosis Society; and International Association for the Study of Obesity. Circulation, 2009. Vol. 20, pp. 1640-1645. doi: 10.1161/Circulationaha.109.192644. Epub 2009 Oct 5.

14. Borodkina D.A. i dr. Raspredelenie zhirovyh otlozhenij: razgadka kazhushchegosya paradoksa ozhireniya $\mathrm{v}$ kardiologii? [ Body fat distribution: the answer to the apparent paradox of obesity in cardiology?]. Ozhirenie i metabolizm [Obesity and metabolism]. 2017. Vol. 14. № 2, pp. 3-8

15. Goncharova N.G. i dr. Rasprostranennost' faktorov riska arterial'noj gipertonii sredi korennogo naseleniya Respubliki Mordoviya [Prevalence of risk factors of hypertension among the indigenous population of the Republic of Mordovia ]. Kazanskij medicinskij zhurnal [Kazan Medical Journal]. 2010. Vol. 91, №, pp. 97-101.

16. Polyakov L.M. i dr. Lipidnyj spektr syvorotki krovi u predstavitelej korennogo i prishlogo naseleniya yamalo-neneckogo avtonomnogo okruga [Serum lipid spectrum of indigenous and alien population of yamalo-nenets autonomous okrug]. Sibirskij nauchnyj medicinskij zhurnal [Siberian Scientific Medical Journal]. 2015. Vol.6, pp. 67-69. 
17. Ogarkov M.Yu. i dr. Rasprostranennost' komponentov metabolicheskogo sindroma u korennogo i nekorennogo naseleniya Gornoj SHorii [The metabolic syndrome main components prevalence of aboriginal and non-aboriginal population of gornaya shoria ]. Byulleten' SO RAMN [Bulletin SB RAMS]. 2004. №1. pp. 108-111.

18. Odegaard J.I., Chawla A. Pleiotropic Actions of Insulin Resistance and Inflammation in Metabolic Homeostasis. Science, 2013. Vol. 339, № 6116, pp. 172177. doi: $10.1126 /$ science. 1230721 .

19. Cut off values for abdominal obesity as a criterion of metabolic syndrome in an ethnic Kyrgyz population (Central Asian region). Cardiovasc Diabetol. 2012 Feb 22;11:16. doi: 10.1186/1475-2840-11-16.

20. Klimova T.M., Fedorova V.I., Baltahinova M.E. Kriterii ozhireniya dlya identifikacii metabolicheskih faktorov riska u korennogo sel'skogo naseleniya YAkutii [Obesity criteria for identifying metabolic risk factors among indigenous rural population of Yakutia]. Sibirskij medicinskij zhurnal [Journal of siberian medical sciences]. 2012. Vol. 115, №. 8, pp. 110-113.

21. Zaher Z.M.M. et al. Optimal cut-off levels to define obesity: body mass index and waist circumference, and their relationship to cardiovascular disease, dyslipidaemia, hypertension and diabetes in Malaysia. Asia Pacific journal of clinical nutrition. 2009. Vol, 18, №. 2, pp. 209-216.

22. Oka R. et al. Reassessment of the cutoff values of waist circumference and visceral fat area for identifying Japanese subjects at risk for the metabolic syndrome. Diabetes research and clinical practice. 2008. Vol. 79, №. 3, pp. 474-481.

23. Delavari A. et al. First nationwide study of the prevalence of the metabolic syndrome and optimal cutoff points of waist circumference in the Middle East: the national survey of risk factors for noncommunicable diseases of Iran. Diabetes care. 2009. Vol. 32, №. 6, pp. 1092-1097.

24. Berseneva O.A., Ageeva E.S. Osobennosti metabolicheskogo sindroma v period menopauzy u zhenshchin Hakasii [Peculiarities of metabolic syndrome during menopause in women of khakasia]. Sibirskoe medicinskoe obozrenie [Siberian medical review]. 2014. № 5, pp. 71-74.

\section{ДАННЫЕ ОБ АВТОРАХ}

Берсенёва Ольга Александровна, преподаватель кафедры фундаментальной медицины и гигиены Федеральное государственное бюджетное образовательное учреждение выстего образования «Хакасский государственный университет им. Н.Ф. Катанова» 
ул. Ленина, 90, Республика Хакасия, г. Абакан, 655000, Российская Федераиия mylanya2010@yandex.ru

Агеева Елизавета Сергеевна, доцент, доктор медицинских наук, профессор кафедры фундаментальной медицины и гигиены Федеральное государственное бюджетное образовательное учреждение высшего образования «Хакасский государственный университет им. Н.Ф. Катанова» ул. Ленина, 90, Республика Хакасия, г. Абакан, 655000, Российская Федераиия

ageevaeliz@rambler.ru

\section{DATA ABOUT THE AUTHORS}

Berseneva Olga Alexandrovna, Teacher of the Department of Fundamental Medicine and Hygiene

Katanov Khakass State University

90, Lenin Str., Republic of Khakassia, Abakan, 655000, Russian Federation

mylanya2010@yandex.ru

SPIN-code: $7310-0675$

ORCID: 0000-0001-5622-2364

ResearcherID: C-6356-2018

Ageeva Elizaveta Sergeevna, Associate Professor, Doctor of Medical Sciences, Professor of the Department of Fundamental Medicine and Hygiene Katanov Khakass State University

90, Lenin Str., Republic of Khakassia, Abakan, 655000, Russian Federation

ageevaeliz@rambler.ru

SPIN-code: 9958-7298

ORCID: 0000-0003-3770-2965

ResearcherID: Q-4704-2016 